\title{
Diffuse Facial Hyperpigmentation as a Presenting Sign of Lupus Erythematosus: Three Cases and Review of the Literature
}

\author{
Shir Azrielant ${ }^{a} \quad$ Eran Ellenbogen $^{\mathrm{a}, \mathrm{b}}$ Alon Peled $^{\mathrm{a}, \mathrm{b}}$ \\ Valentina Zemser-Werner ${ }^{c}$ Liat Samuelov ${ }^{a}$ b Eli Sprecher ${ }^{a}$ b \\ Mor Pavlovsky ${ }^{a}$ \\ ${ }^{a}$ Division of Dermatology, Tel Aviv Sourasky Medical Center, Tel Aviv, Israel; 'bSackler Faculty \\ of Medicine, Tel Aviv University, Tel Aviv, Israel; 'Department of Pathology, Tel Aviv Sourasky \\ Medical Center, Tel Aviv, Israel
}

\section{Keywords}

Hyperpigmentation · Lupus erythematosus · Photosensitivity · Connective tissue disorders

\begin{abstract}
Lupus erythematosus (LE) is an autoimmune disorder commonly affecting the skin; cutaneous lesions may indicate systemic involvement, warranting further evaluation. Photosensitivity, which may result in hyperpigmentation, is a well-known feature of the disease. In contrast, the prevalence of primary hyperpigmentation as a presenting sign of LE is not well established. Here, we compare 3 unique cases of diffuse facial hyperpigmentation as the primary manifestation of LE (cutaneous or systemic) and review previously reported cases. Our data highlight the need for considering LE in the differential diagnosis of facial hyperpigmentation and substantiate the importance of this unique lupus variant in early diagnosis and patient evaluation.
\end{abstract}

(C) 2021 The Author(s).

Published by S. Karger AG, Basel

\section{Introduction}

Cutaneous lupus erythematosus (LE; CLE) is a chronic autoimmune disease that commonly impairs patients' quality of life and indicates systemic involvement. Cutaneous lesions of LE were originally subdivided into acute, subacute, and chronic lesions [1]. The former is most commonly characterized by the typical malar ("butterfly") rash, while papulosquamous lesions and discoid rash are more characteristic of subacute and chronic forms [2]. 
However, many rare variants of CLE have been reported, including LE tumidus, perfundus, and hypertrophicus [3]. Recognition of these atypical variants is of high importance, given the role played by dermatologists in the initial diagnosis of primary CLE lesions. Here, we present 3 cases of facial hyperpigmentation eventually diagnosed as CLE or systemic LE and review the literature of previously reported similar cases aiming to clarify the prevalence of facial hyperpigmentation as the presenting sign of LE.

\section{Case Reports}

Demographic, clinical, and histopathological data regarding all cases are presented in Table 1.

Case 1

A 56-year-old man of Indian origin presented with an 8-month history of hyperpigmented, slightly pruritic facial lesions, previously treated as postinflammatory hyperpigmentation with Kligman formulation [4] and topical steroids without improvement. He had no systemic symptoms. The patient's past medical history was unremarkable, and he did not receive any chronic medications. He had been using facial cosmetic products for several years prior to rash appearance.

On physical examination, the patient had skin type IV according to Fitzpatrick classification [5] and presented with hyperpigmented scaly thin plaques over the forehead, temples, and cheeks (Fig. 1a, b). A punch biopsy obtained from the patient's forehead showed a superficial and deep perivascular, vacuolar/lichenoid interface dermatitis with thin epidermis (Fig. 2a). Alcian blue stain was weakly positive, and CD-123 stain, a marker for plasmacytoid monocytes often detected in LE [6], was positive (Fig. 2b).

Further evaluation revealed positive antinuclear antibodies (ANA) at titers of 1:640 with a speckled pattern and positive anti-Ro antibodies. The patient had slightly abnormal coagulation studies (PT 16.8, PTT 38.3, INR 1.2) with negative Coombs' test. Urine analysis showed small amounts of protein (11-50 mg/dL) without casts. Other laboratory tests were unremarkable.

Patch tests using the European standard and cosmetics series were positive for thiuram mix, a finding that was deemed insignificant in the absence of relevant exposure. Based on the histologic findings and autoimmune serology, the patient was diagnosed with a hyperpigmented variant of CLE. Systemic treatment with hydroxychloroquine (200 mg, twice daily) was initiated with slight improvement following 6 months of treatment.

Case 2

A 52-year-old man of Sephardic Jewish origin presented with a 3-year history of hyperpigmented asymptomatic rash involving the face. He had no accompanying systemic symptoms. The rash had previously been clinically diagnosed as a hyperpigmented form of demodecidosis and had been treated with ivermectin cream without improvement. His past medical history was remarkable for active smoking (30 pack-years) and hypertension. An angiotensin-converting enzyme (ACE) inhibitor treatment for the latter was initiated 1 year prior to his admission.

On physical examination, the patient had skin type III-IV according to Fitzpatrick classification. Hyperpigmented poorly demarcated thin plaques were noted over his forehead and cheeks (Fig. 1c, d). Skin biopsies obtained from hyperpigmented patches revealed interface dermatitis, and perivascular and periadnexal infiltrates with a thickened basement membrane and melanophages (Fig. 2c). Direct immunofluorescence (DIF) was positive for immunoglobulin

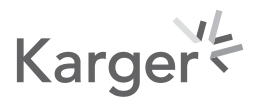


Case Reports in Dermatology
Case Rep Dermatol 2021;13:263-270 DOI: 10.1159/000515732

(c) 2021 www.karger.com/cde

Azrielant et al.: Hyperpigmented Variant of Lupus Erythematosus

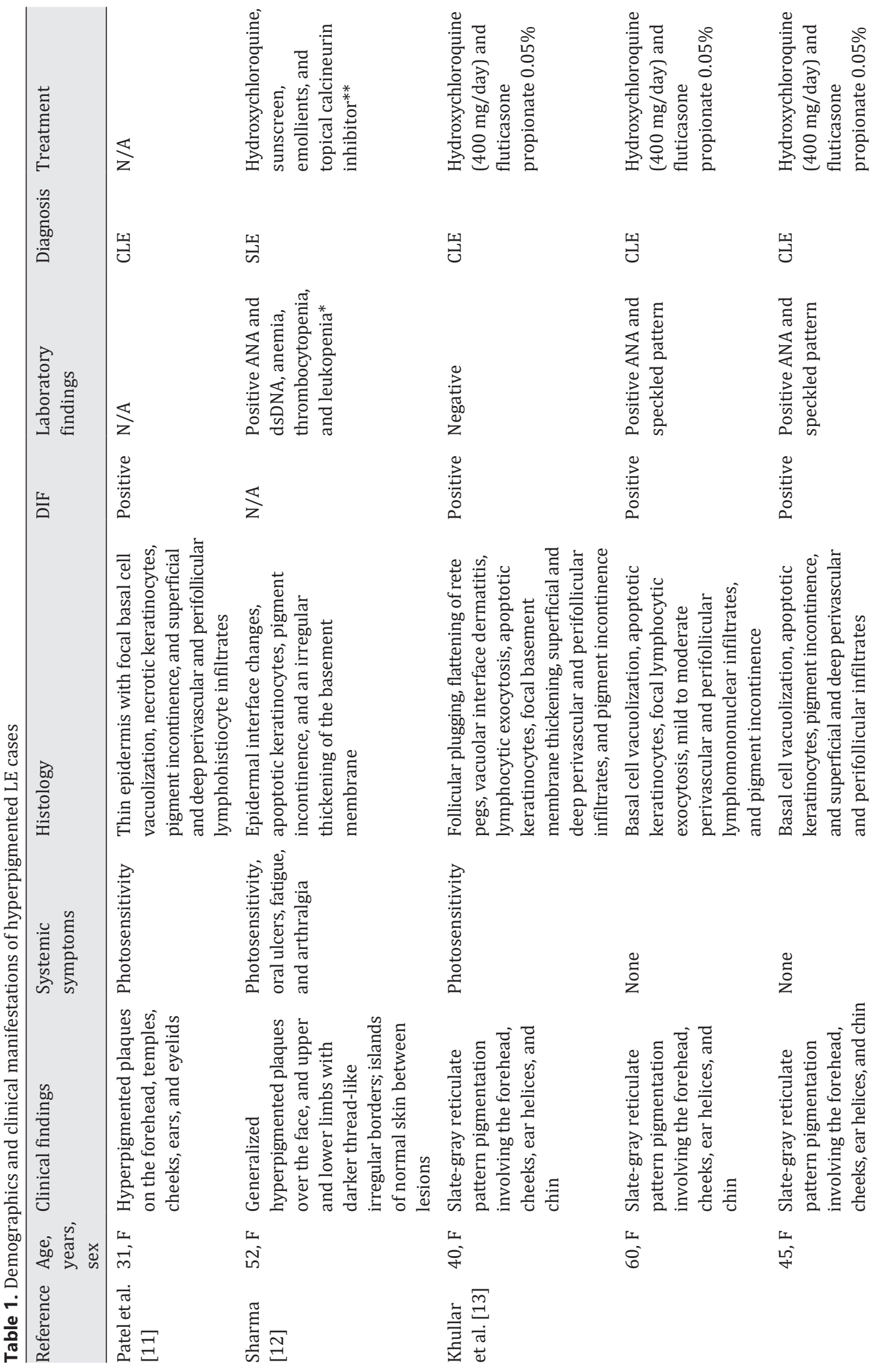


Case Reports in Dermatology
Case Rep Dermatol 2021;13:263-270

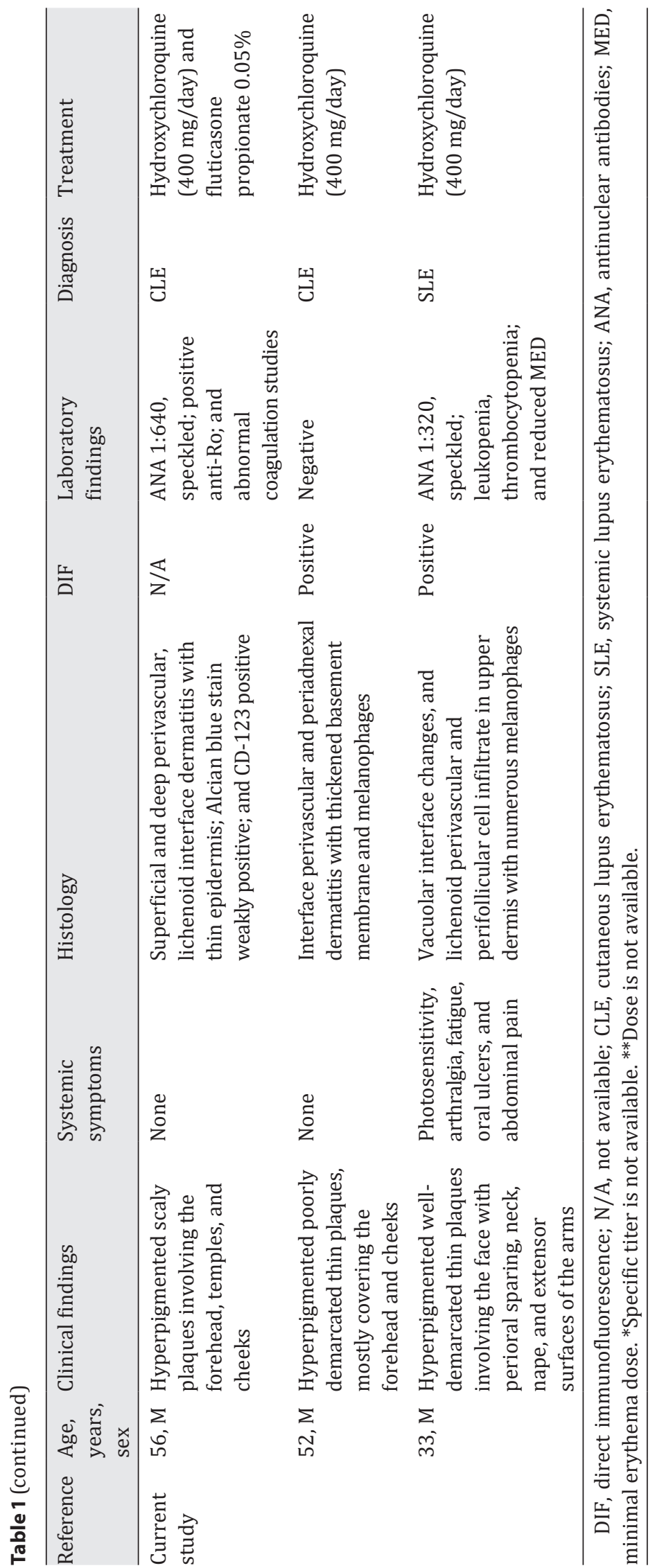




\section{Case Reports in Dermatology}

\begin{tabular}{l|l}
\hline Case Rep Dermatol 2021;13:263-270 \\
\hline DOI: 10.1159/000515732 & $\begin{array}{l}\text { @ 2021 The Author(s). Published by S. Karger AG, Basel } \\
\text { www.karger.com/cde }\end{array}$ \\
\hline
\end{tabular}

Azrielant et al.: Hyperpigmented Variant of Lupus Erythematosus
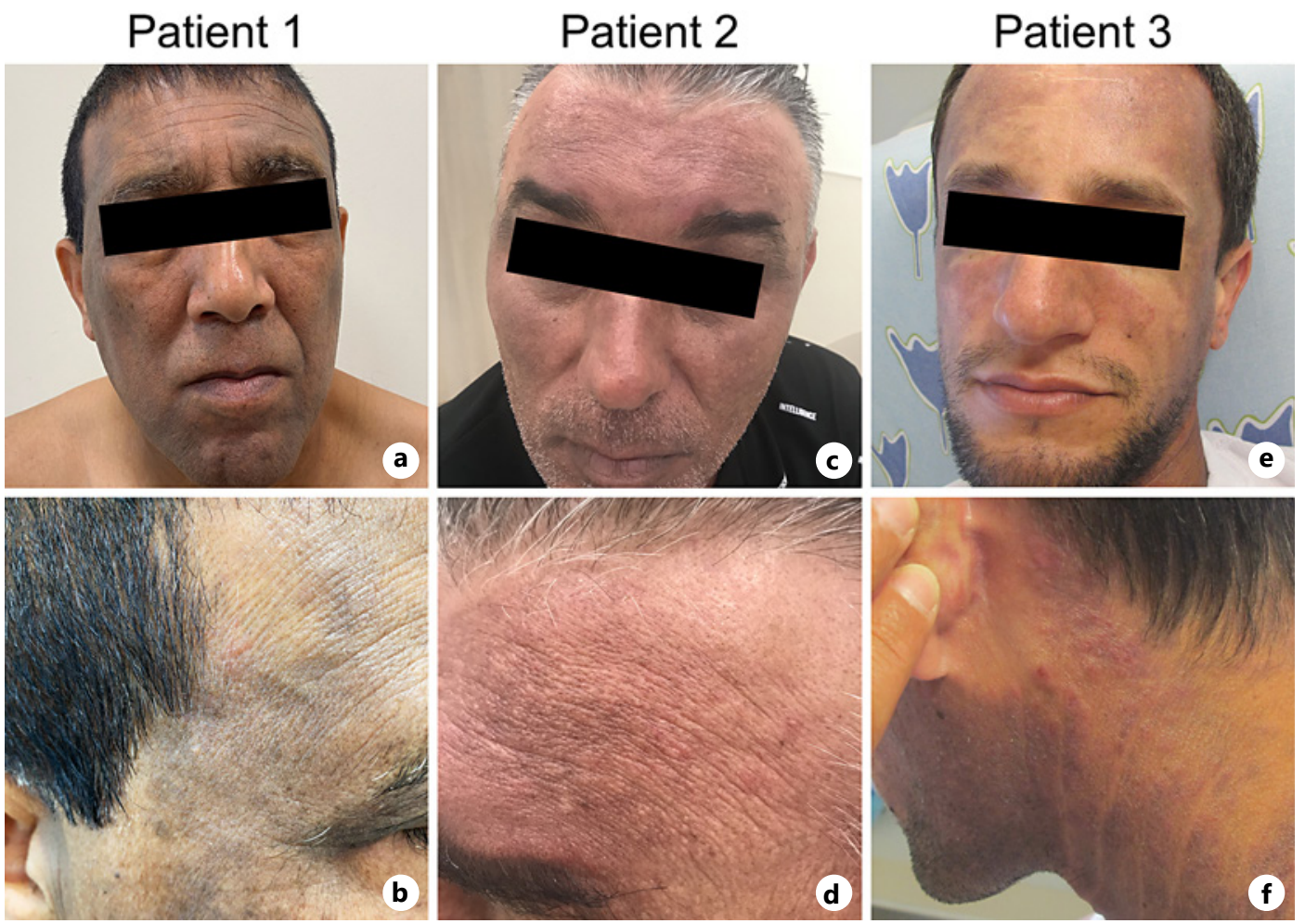

Fig. 1. Clinical features. Hyperpigmented plaques over the forehead, temples, and cheeks in patient 1 (a, b); poorly demarcated hyperpigmented thin plaques over the face in patient 2 (c, d); hyperpigmented thin plaques over the neck, nape, periauricular area, and face in patient $3(\mathbf{e}, \mathbf{f})$. Note perioral sparing (e).

Patient 1
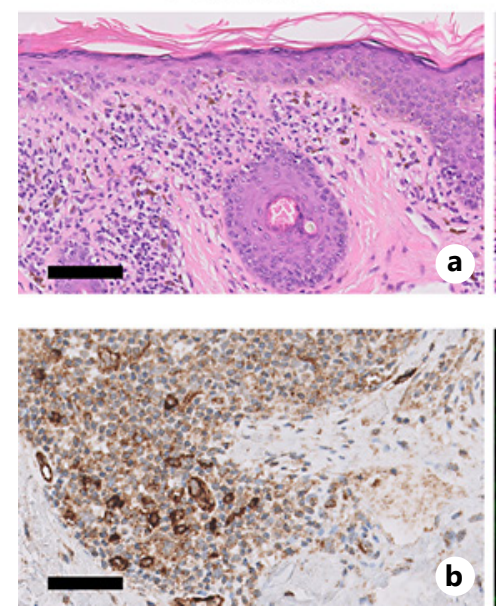

Patient 2

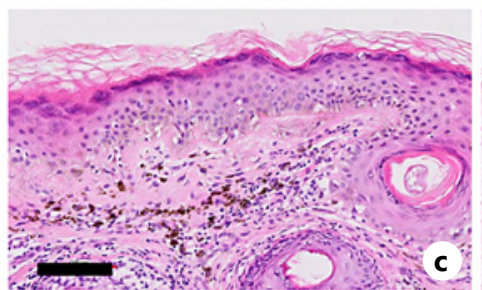

Patient 3
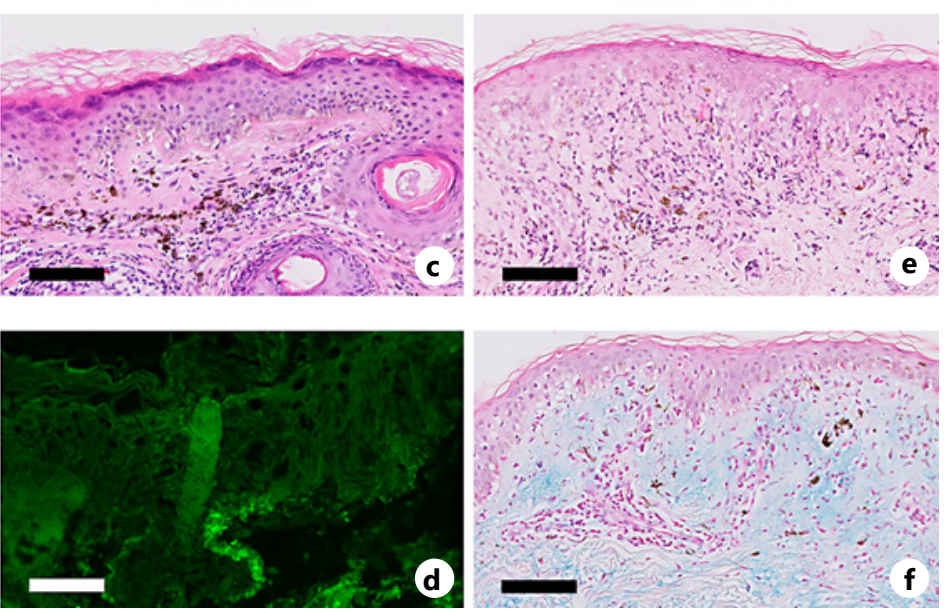

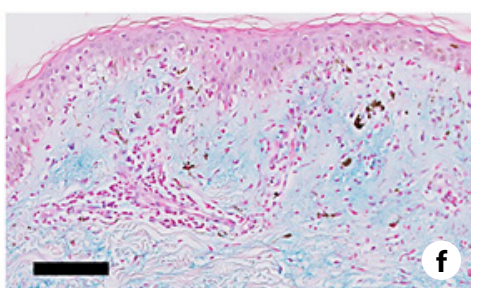

Fig. 2. Histological features of patients. Skin biopsy of patient 1 showing superficial and deep perivascular, lichenoid interface dermatitis (H\&E stain) (a); inflammatory infiltrate in the same biopsy stains positive for CD123 (b); skin biopsy of patient 2 showing interface perivascular and peri-adnexal dermatitis with thickened basement membrane and melanophages (H\&E stain) (c); DIF of patient 2 demonstrating weak granular deposition of IgM at the DEJ ("lupus band") (d); skin biopsy of patient 3 showing vacuolar interface changes, and lichenoid perivascular and perifollicular cell infiltrates in the upper dermis with numerous melanophages (H\&E stain) (e); the same biopsy demonstrates positive Alcian blue staining (f). Scale bars $=100 \mu \mathrm{M}$. H\&E, hematoxylin and eosin; DIF, direct immunofluorescence; IgM, immunoglobulin M; DEJ, dermal-epidermal junction. 
M (Fig. 2d) and C3 with a weak granular pattern at the dermal-epidermal junction typical for "lupus-band." Laboratory tests including autoimmune serology were unremarkable. Patch tests with the European standard and cosmetics series were positive for chlorhexidine, but the patient denied any history of exposure to this agent.

Based on the histologic findings and positive lupus band on DIF, the patient was diagnosed with hyperpigmented CLE without systemic involvement. Treatment with hydroxychloroquine was initiated with minimal clinical improvement.

Case 3

A 33-year-old man of Jewish origin presented with an 18-month history of hyperpigmented rash that involved sun-exposed areas including the face, neck, nape, and arms. The rash was mildly pruritic and was accompanied by intermittent pain of the knees and elbows, fatigue, occasional abdominal pain, and oral ulcers. The patient had significant occupational sun exposure that resulted in gradual worsening of his rash. This posed a great limitation to his daily work routine.

His past medical history was positive for heavy smoking (17 pack-years). He denied any chronic illnesses or medication use.

On physical examination, the patient had skin type III according to Fitzpatrick classification. Hyperpigmented well-demarcated thin plaques were evident over the neck, nape, and extensor surfaces of the arms and face, with typical sparing of skin folds (Fig. 1e, f).

Three skin biopsies obtained from hyperpigmented patches revealed vacuolar interface changes, and lichenoid perivascular and perifollicular cell infiltrates in the upper dermis with numerous melanophages (Fig. 2e). DIF from lesional skin demonstrated deposition of immunoglobulin $\mathrm{M}$ and $\mathrm{C} 3$ in a linear granular pattern at the dermal-epidermal junction. Alcian blue stain was positive (Fig. 2f).

Laboratory tests revealed mild leukopenia $(2,700$ cells $/ \mu \mathrm{L})$ and mild thrombocytopenia of $(140,000$ cells $/ \mu \mathrm{L})$. Autoimmune serology revealed positive ANA, with a titer of 1:320 in a speckled pattern; all other serologies and complement levels were normal. The urine test was normal. The patch test (standard series) was negative, and the photo-provocation test revealed reduced minimal erythema dose.

Based on the abovementioned findings, the patient was diagnosed with systemic LE (SLE) according to the 2012 SLICC criteria. Systemic treatment with hydroxychloroquine (200 mg, twice daily) was initiated with great improvement in his symptoms.

\section{Discussion}

LE is a multisystem, chronic autoimmune disease with potentially serious complications. Cutaneous manifestations in LE include LE-nonspecific and LE-specific skin lesions, the latter being typically associated with vacuolar interface dermatitis on histology [1]. CLE is present in over $75 \%$ of patients at some point during the course of SLE and is the second most frequent presenting symptom following joint involvement [7]. Since cutaneous lesions may be the presenting sign of the disease, the general dermatologist should be familiar with typical and atypical skin lesions of LE.

In this study, we report 3 male patients who presented with facial hyperpigmentation as initial presentation of CLE or systemic LE. Hyperpigmentation is a well-recognized sequelae of LE. Previous studies from Pakistan [8], India [9], and the USA [10] have shown that hyperpigmentation is present in $37.5,25$, and $8.4 \%$ of LE patients, respectively, with higher prevalence observed in patients with darker complexion. However, these studies did not differentiate between the possible causes of hyperpigmentation in LE patients: primary manifestation

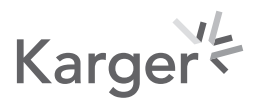


of LE, postinflammatory changes secondary to active inflammation or photosensitization, or medications (e.g., antimalarial medications or methotrexate).

To the best of our knowledge, to date, only 5 cases of primary hyperpigmented LE have been described previously in the literature [11-13] (Table 1). The series reported by Boyd [14] reviewed eleven cases of isolated pigmented head and neck skin lesions in elderly patients; although histology in these cases demonstrated interface dermatitis, serologies (except for 2 cases positive for ANA) and DIF were either normal or absent, so it is difficult to classify those cases, which were therefore not included in the literature review presented here.

The 3 patients described in the present study and the 5 previously reported cases (Table 1) exemplify the distinctive features of this apparently rare form of LE. First, this variant predominates in patients with darker skin $(80 \%$ of reported cases occurred in the Indian population) and in patients in their fourth or fifth decade of life. Second, this clinical variant, like other types of CLE, favors the face, dorsal hands, and other sunexposed areas. Two cases (one of the patients presented here and one previously reported) presented with systemic involvement (Table 1), underscoring the importance of systemic evaluation in this rare subset of the disease. In contrast to previously reported cases of hyperpigmented LE who were female cases and showed clinical improvement with antimalarial medications, the 3 patients reported here were males and only one patient demonstrated significant improvement following several months of treatment with hydroxychloroquine.

Like many subtypes of CLE, the pathogenesis of the hyperpigmented LE remains to be delineated. We hypothesize that the interface dermatitis that leads to pigment incontinence, especially in darkly pigmented individuals, is responsible for the development of hyperpigmentation.

Although ACE inhibitors were previously linked to various manifestations of CLE [15], patient 2 started his antihypertensive treatment only 2 years after the initial presentation of his symptoms. Therefore, we did not attribute his disease to ACE inhibitor usage, and the treatment has been continued.

In summary, the present study underscores the need to include LE in the differential diagnosis of facial hyperpigmentation. Larger and longitudinal studies are needed to determine the course, prognosis, and optimal treatment of this rare variant of a common disease.

\section{Statement of Ethics}

All patients have given written informed consent to publication of their case details and any accompanying images. The paper is exempt from ethical committee approval as this is a retrospective case series without any therapeutic intervention.

\section{Conflict of Interest Statement}

The authors have no conflicts of interest to declare.

\section{Funding Sources}

The authors did not receive any funding.

\section{Karger'}




\section{Case Reports in Dermatology}

\begin{tabular}{l|l}
\hline Case Rep Dermatol 2021;13:263-270 \\
\hline DOI: 10.1159/000515732 & $\begin{array}{l}\text { @ 2021 The Author(s). Published by S. Karger AG, Basel } \\
\text { www.karger.com/cde }\end{array}$ \\
\hline
\end{tabular}

Azrielant et al.: Hyperpigmented Variant of Lupus Erythematosus

\section{Author Contributions}

All authors fulfill the ICMJE criteria for authorship. Shir Azrielant: concept, acquisition, interpretation, design, and manuscript preparation; Eran Ellenbogen: acquisition, interpretation, editing, and review; Alon Peled: design, editing, and review; Valentina Zemser-Werner: acquisition review; Liat Samuelov: interpretation and review; Eli Sprecher: interpretation, editing, and review; and Mor Pavlovsky: concept, acquisition, interpretation, editing, and review.

\section{References}

1 Gilliam JN, Sontheimer RD. Distinctive cutaneous subsets in the spectrum of lupus erythematosus. J Am Acad Dermatol. 1981;4:471-5.

2 Okon LG, Werth VP. Cutaneous lupus erythematosus: diagnosis and treatment. Best Pract Res Clin Rheumatol. 2013;27:391-404.

3 Pramatarov KD. Chronic cutaneous lupus erythematosus: clinical spectrum. Clin Dermatol. 2004;22:113-20.

4 Kligman AM, Willis I. A new formula for depigmenting human skin. Arch Dermatol. 1975;111:40-8.

5 Fitzpatrick TB. The validity and practicality of sun-reactive skin types I through VI. Arch Dermatol. 1988;124: 869-71.

6 Farkas L, Beiske K, Lund-Johansen F, Brandtzaeg P, Jahnsen FL. Plasmacytoid dendritic cells (natural interferon- alpha/beta-producing cells) accumulate in cutaneous lupus erythematosus lesions. Am J Pathol. 2001; 159:237-43.

7 Obermoser G, Sontheimer RD, Zelger B. Overview of common, rare and atypical manifestations of cutaneous lupus erythematosus and histopathological correlates. Lupus. 2010;19:1050-70.

8 Kapadia N, Haroon TS. Cutaneous manifestations of systemic lupus erythematosus: study from Lahore, Pakistan. Int J Dermatol. 1996;35:408-9.

9 George R, Mathai R, Kurian S. Cutaneous lupus erythematosus in India: immunofluorescence profile. Int J Dermatol. 1992;31:265-9.

10 Dubois EL, Tuffanelli DL. Clinical manifestations of systemic lupus erythematosus. computer analysis of 520 cases. JAMA. 1964;190:104-11.

11 Patel AB, Kubba R, Kubba A. Clinicopathological correlation of acquired hyperpigmentary disorders. Indian J Dermatol Venereol Leprol. 2013;79:367-75.

12 Sharma R. Generalized pigmented lupus erythematosus: a new clinical subset? J Pigment Disord. 2016;3.

13 Khullar G, De D, Narang T, Saikia UN, Saikia B, Handa S. Pigmented macular variant of chronic cutaneous lupus erythematosus: an under-recognized subset in dark skin. Clin Exp Dermatol. 2017;42:793-5.

14 Boyd AS. Localized chronic cutaneous lupus erythematosus masquerading as pigmented lesions: a new clinical subset? Lupus. 2006;15:292-5.

15 Marzano AV, Vezzoli P, Crosti C. Drug-induced lupus: an update on its dermatologic aspects. Lupus. 2009;18: 935-40. 